\title{
A Visualizing Study of Blister Initiation Behavior by Gas Decompression
}

\author{
Atsushi Koga $^{1)^{*}}$, Tadahisa Yamabe $^{1)}$, Hiroyuki Sato ${ }^{1)}$, Kenichi Uchida $^{1)}$, Junichi Nakayama ${ }^{1)}$, \\ Junichiro Yamabe ${ }^{2,4)}$ and Shin Nishimura ${ }^{3,4)}$ \\ ${ }^{1)}$ NOK Corporation \\ 4-3-1 Tsujido-shinmachi, Fujisawa, Kanagawa 251-0042, Japan \\ ${ }^{2}$ International Research Center for Hydrogen Energy, Kyushu University \\ 744 Motooka, Nishi-ku, Fukuoka 819-0395, Japan \\ ${ }^{3)}$ Department of Mechanical Engineering, Faculty of Engineering, Kyushu University \\ 744 Motooka, Nishi-ku, Fukuoka 819-0395, Japan \\ ${ }^{4)}$ The Research Center for Hydrogen Industrial Use and Storage, \\ National Institute of Advanced Industrial Science and Technology \\ 744 Motooka, Nishi-ku, Fukuoka 819-0395, Japan \\ *Corresponding author: koga199@nok.co.jp
}

\begin{abstract}
( Manuscript received 26 December 2011; accepted 28 October 2012; published 31 January 2013 )
( Presented at Symposium S8: Hydrogen Tribology for Future Energy in the International Tribology Conference Hiroshima 2011 )
\end{abstract}

\begin{abstract}
This study presents the effects of the type of polymer and gas on blister fracture in terms of visualizing O-ring behavior under high-pressure gas. To visualize blisters (internal cracks) by optical microscopy, transparent EPDM and VMQ O-rings were molded, and a special viewable high-pressure vessel with a glass viewport was developed. The O-ring specimens were exposed to high-pressure hydrogen, helium, and nitrogen gases at $10 \mathrm{MPa}$ under room temperature, $25^{\circ} \mathrm{C}$, after which these gases were rapidly decompressed for 0.3 seconds. The blister fracture occurred in EPDM but not in VMQ even though the two materials had the same Young's modulus. It is presumed by AFM observation that the difference in microstructure at sub-micrometer level between EPDM and VMQ influenced their blister initiation. The blister damage of the transparent EPDM O-ring in nitrogen gas was the most serious, while that in the helium gas was the slightest. The reason why the blister damage of the O-ring in the helium gas was the slightest is considered to be because solute helium gas diffused out from the O-ring after decompression faster than the other gases.
\end{abstract}

Keywords: rubber, O-ring, blister, high-pressure gas, decompression failure, decompression rate, atomic force microscopy

\section{Introduction}

The depletion of fossil fuels and global warming are social problems faced today. To resolve these issues, the realization of a hydrogen society which makes good use of the high energy availability of hydrogen utilizing fuel cell systems is sought. However, in order to realize this hydrogen society, there is a need to ensure the safety of hydrogen energy related systems, and to fully understand the effects of hydrogen on the mechanical, physical, and chemical properties of metallic and polymeric materials used for hydrogen energy related equipment. FCVs (Fuel Cell Vehicle) are mounted with various hydrogen energy related equipment such as built-in hydrogen gas transport comprising of hydrogen tank, valves, pipes, etc., hydrogen gas supply equipment made up of receptacle, etc. which supplies hydrogen into the FCV, and safety equipment such as pressurization prevention safety device. In all of these equipments, rubber material is applied as seals of O-rings, gaskets, and packing. For this reason, the assurance of the safety and reliability of rubber seals is vital in the building of the hydrogen society.

In these rubber seal materials, it is necessary to maintain sealing function in high-pressure gas environments or pressurization/decompression gas cycles. The high-pressure gas cycle causes internal cracks called blister which reduce the sealing durability of rubber. To date, blister damage has been reported for numerous gases and polymers [1-3]. The authors have also confirmed the occurrence of blister damage in high-pressure hydrogen gas and clarified the effects of fillers [4] and environmental factors [5] on blister occurrence. However, much about blister occurrence and growth mechanisms remains unclear. Yamabe et al. [6] are reviewing related behavior in the nano and micron orders from the formation of gas bubbles which cause 
blisters using atomic force microscopes (AFM) and acoustic emission method (AE). In investigations with hydrogen gas-exposed EPDM rubber specimens (Ethylene Propylene Diene, M-class) which is a type of synthetic rubber under the conditions of $25^{\circ} \mathrm{C}$ and 10 $\mathrm{MPa}$, they are proposing a multi-scale fracture process where gas bubbles are formed starting from sub-micron-size low strength areas, then growing into micro to millimeter sized blisters. Here, gas bubble is defined as submicron to micron-size cavities that are difficult to observe under the optical microscope, and blisters as cavities larger than micron-size which can be observed under the optical microscope. The same definitions were applied in the study. As the blister occurrence limit pressure can be evaluated quantitatively from the energy balance with lower limit fracture energy, which is a macroscopic fracture mechanics parameter, blisters can be said to be a type of damage accompanied by macroscopic molecular chain breakage independent of micro-heterogeneous structures. However, it is unclear if such fracture process works for other types of polymers and gases, and further verifications are required.

In this study, we used transparent EPDM rubber and silicone rubber (VMQ; Vinyl Methyl polysiloxane,
Q-class) which is a type of synthetic rubber to visualize blisters with an optical microscope. We also developed a high-pressure gas exposure container enabling visualization of internal changes of the rubber O-ring caused by pressurization/decompression of high-pressure gas. Using hydrogen, helium, and nitrogen as the high-pressure test gases, the effects of different types of polymers and gas species on blister initiation at rubber O-rings were investigated.

\section{Experimental Methods}

\subsection{Rubber material and specimen}

To visualize blisters occurring inside rubber, transparent rubber material was prepared. As the base polymers, EPDM and VMQ were selected and cured with peroxides. For the EPDM rubber composition, $2 \mathrm{phr}$ of dicumyl peroxide was added to EPDM polymer of the ENB (5-ethylidene - 2-norbornene) type containing 56 wt $\%$ ethylene. The term "phr" stands for "parts per hundred rubber" to represent the parts by weight per 100 parts by weight of polymer. For the VMQ rubber composition, $0.3 \mathrm{phr}$ of tert - butyl cumyl peroxide was added to VMQ polymer with excellent transparency. Both were then press molded for four minutes at $180^{\circ} \mathrm{C}$,

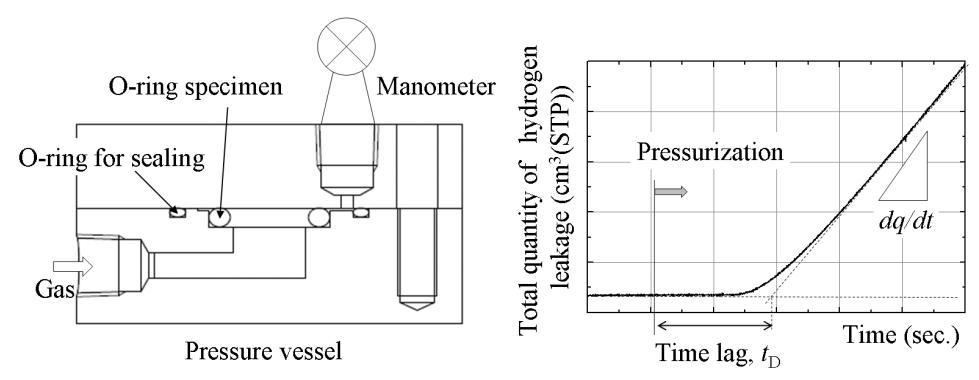

(a-1) Schematic illustration of permeation test (a-2) Example of permeation curve

(a) Gas permeation test

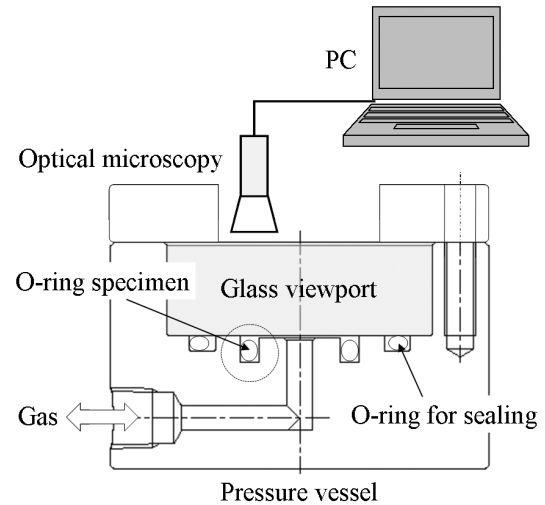

(b-1) Schematic illustration of visualization of blister

(b) Visualization of blister

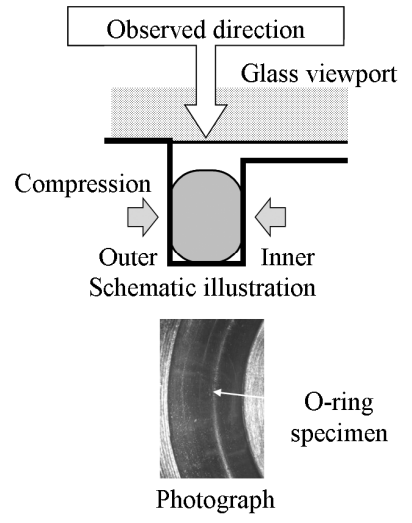

(b-2) Details of installation of O-ring specimen

Fig. 1 Experimental equipment: (a) Gas permeation test, (a-1) Schematic illustration of permeation test, (a-2) Example of permeation curve; (b) Visualization of blister, (b-1) Schematic illustration of visualization of blister, (b-2) Details of installation of O-ring specimen 
and thermally post cured for four hours at $120^{\circ} \mathrm{C}$ to form test sheets (thickness $2 \mathrm{~mm}$ ) and O-ring specimens (wire diameter $\phi 3.53 \mathrm{~mm}$, inner diameter $\phi 11.9 \mathrm{~mm})$. The hardness of both the EPDM and VMQ was 50 in JIS durometer hardness type A. The Young's modulus obtained in tensile tests using JIS K6251 No. 3 dumbbells was $2 \mathrm{MPa}$ for both EPDM and VMQ, and breaking elongation was $150 \%$ (EPDM) and 700\% (VMQ) respectively. Compared to EPDM, VMQ has the same Young's modulus, but greater break elongation.

\subsection{Gas permeation test}

Figure 1(a-1) shows the gas permeation test using O-rings. By using O-rings as the specimens, gas permeability at high pressure which could not be measured with rubber sheets can be evaluated. In this study, hydrogen, helium, and nitrogen were used as the test gases. The O-ring specimens were then installed in flat face static sealing for internal pressure with a squeeze rate of $16 \%$, gas pressurized to $10 \mathrm{MPa}$ was supplied to the inner circumference of the O-rings. As the pressure at the outer circumference was atmospheric pressure $(0.1 \mathrm{MPa})$, permeation tests were conducted at room temperature $\left(25^{\circ} \mathrm{C}\right)$ at about $10 \mathrm{MPa}$ pressure difference with the internal pressure. Figure 1(a-2) shows the relation between the cumulative amount, $q$ of hydrogen permeating through the rubber and leaking from the outer circumference under constant pressure and temperature and time, $t$ (gas permeation curve : $q-t$ curve). The $q-t$ curve in the normal state was approximated by a straight line, and from the gradient $\mathrm{d} q$ / $\mathrm{d} t$ of the approximated straight line, and the delay time, $t_{\mathrm{D}}$ represented by the intersection between the approximated straight line and $x$ axis, the gas permeation coefficient, gas diffusion coefficient, and gas solubility coefficient can be estimated $[7,8]$.

\subsection{Blister visualization}

Figure 1(b-1) shows the schematic illustration of the pressure vessel used for visualizing the blister phenomenon. Figure 1(b-2) shows the details of the pressure vessel with pressure-proof glass. The O-ring specimen is installed so that it is compressed into the O-ring groove at the squeeze rate of $16 \%$ and does not contact the pressure proof glass. Gas is then applied from the top of the O-ring specimen, and the O-ring specimen is observed from the direction in which it is pressurized under the optical microscope (Keyence VHX-900). Like the permeation test, the O-ring specimen was pressurized at $10 \mathrm{MPa}$ using hydrogen, helium, and nitrogen under room temperature $\left(25^{\circ} \mathrm{C}\right)$. The pressurization retention time was set longer than the delay time $\left(t_{\mathrm{D}}\right)$ obtained from the gas permeation curve so that the gas inside the rubber saturates adequately. After this, the high-pressure gas was rapidly decompressed from $10 \mathrm{MPa}$ to atmospheric pressure in 0.3 seconds to observe the changes inside the rubber during the decompression process.

\subsection{Material structure observation by AFM}

Using an atomic force microscope (AFM), we also observed the sub-micron level structures of EPDM and VMQ. The insides of O-rings unexposed and exposed to gas were removed, and surfaces on which no blisters could be visually seen were finished smoothly using a cryomicrotome (Leica Microsystems EM FC6). For AFM observation, Veeco Dimension 3100 / Nanoscope 5 was used to observe the finished surfaces in room temperature in the tapping mode. Properties of the probe used were resonance frequency of $75 \mathrm{kHz}$, cantilever spring constant of $3 \mathrm{~N} / \mathrm{m}$, and probe radius of less than 15 $\mathrm{nm}$ (all nominal values).

\section{Results and Discussion}

\subsection{Gas permeability}

Figure 2 shows the gas permeation coefficient $Q$, gas diffusion coefficient $D$, and gas solubility coefficient $S$ of EPDM and VMQ measured using hydrogen, helium, and nitrogen at a pressure of $10 \mathrm{MPa}$ and room temperature $\left(25^{\circ} \mathrm{C}\right)$. In this Fig. 2, experiment results are normalized at the permeation, diffusion, and solubility coefficients of hydrogen gas for EPDM.

The gas permeation coefficient was in the order of $\mathrm{H}_{2}$ $\approx \mathrm{He}>>\mathrm{N}_{2}$ for EPDM and $\mathrm{H}_{2} \approx \mathrm{He}>\mathrm{N}_{2}$ for VMQ. The gas permeation coefficients of hydrogen and helium were about 10 times greater for EPDM than nitrogen gas, and about three times greater for VMQ. In addition, regardless of the gas species, the gas permeation coefficient of VMQ was greater than EPDM.

The gas diffusion coefficient was in the order of $\mathrm{He}>$ $\mathrm{H}_{2} \gg \mathrm{N}_{2}$ for both EPDM and VMQ. The diffusion coefficient of hydrogen was about two times smaller than that of helium, and about ten times greater than that of nitrogen. Also, regardless of the gas species, the gas diffusion coefficient of VMQ is ten times greater than EPDM.

The gas solubility coefficient was in the order of $\mathrm{H}_{2}$ $>\mathrm{N}_{2}>\mathrm{He}$ for EPDM and $\mathrm{N}_{2}>\mathrm{H}_{2}>\mathrm{He}$ for VMQ, indicating a different tendency between EPDM and VMQ. Regardless of the gas species, the gas solubility coefficient of VMQ was greater than EPDM.

The gas permeation parameters of EPDM and VMQ obtained with high-pressure gas at pressure of $10 \mathrm{MPa}$

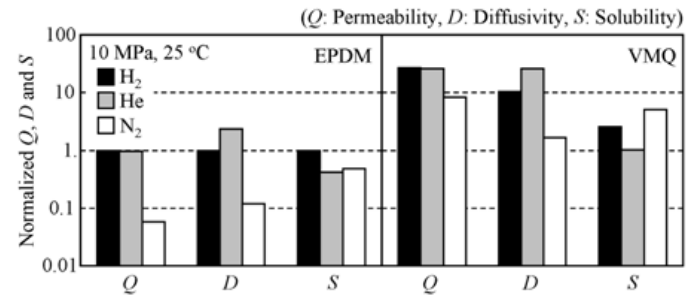

Fig. 2 Normalized gas permeability, gas diffusivity, and gas sohbility of EPDM and VMQ O-ring specimens tested at $10 \mathrm{MPa}$ under $25^{\circ} \mathrm{C}$ 


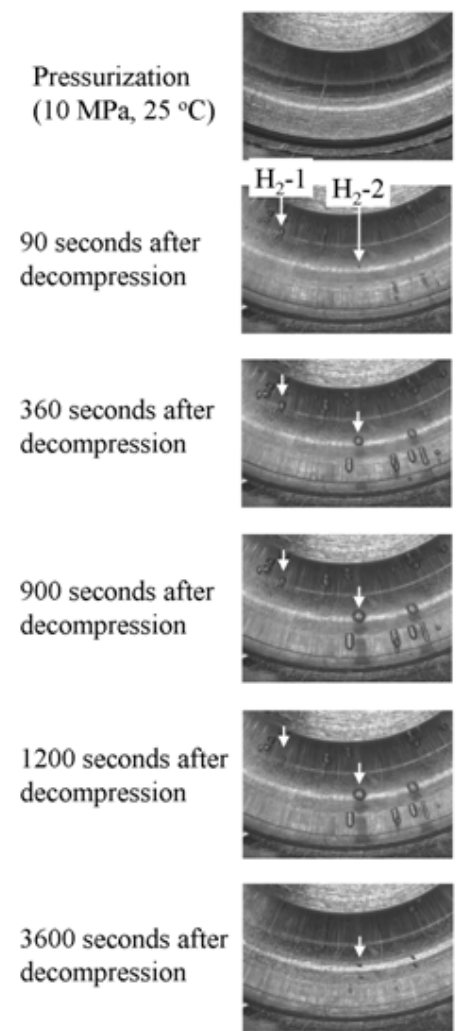

(a) Hydrogen gas
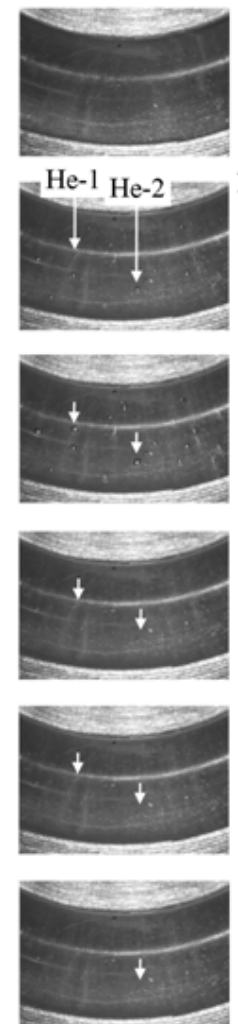

(b) Helium gas
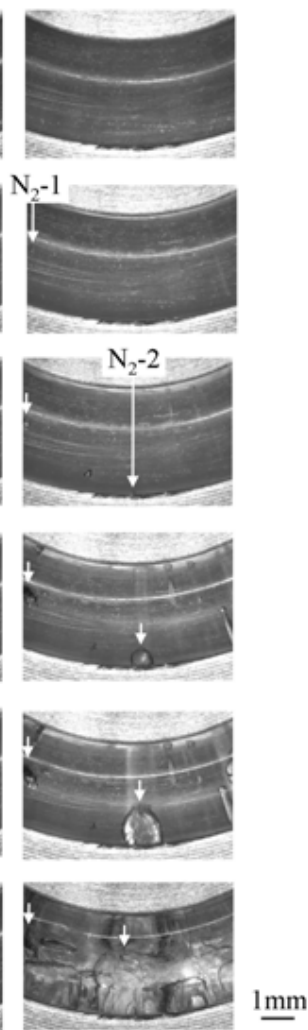

(c) Nitrogen gas

Fig. 3 Blister initiation behavior of EPDM with high-pressure gas decompressions at $10 \mathrm{MPa}$ under $25^{\circ} \mathrm{C}$ : (a) Hydrogen gas, (b) Helium gas, (c) Nitrogen gas

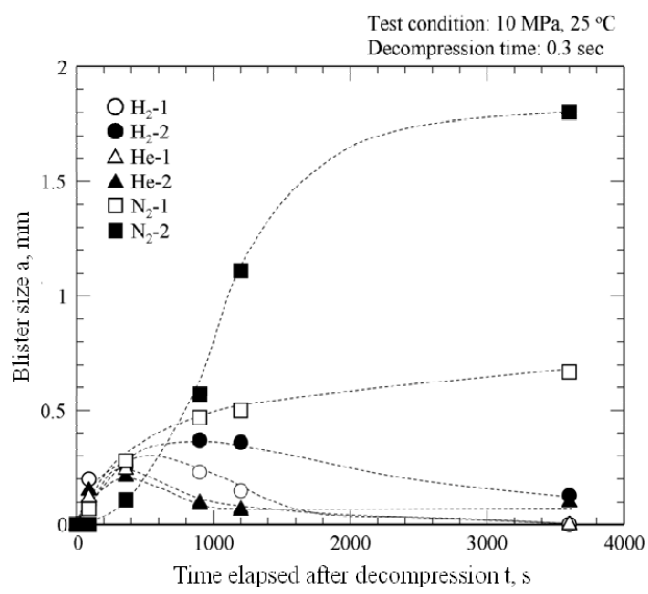

Fig. 4 Relationship between blister size and elapsed time after decompression of EPDM tested in hydrogen, helium, and nitrogen gases at 10 $\mathrm{MPa}$ under $25^{\circ} \mathrm{C}$

using O-ring specimen showed the same tendency as the results measured at the gas pressure of $0.6 \mathrm{MPa}$ using sheet specimens.

\subsection{Blister initiation appearance by visualization}

\subsubsection{EPDM}

Figure 3 shows the results of observation of the insides of O-rings during pressurization and after decompression of EPDM exposed to hydrogen, helium, and nitrogen at a pressure of $10 \mathrm{MPa}$ under an optical microscope. For all gases, blisters were found to occur after decompression, but the blister initiation behavior differed according to the gas species. Figure 4 shows the changes in the size of the two largest blisters with time elapsed after decompression. When the blister initiation behavior was evaluated from the viewpoint of the blister size, the blister size increased in the order of $\mathrm{N}_{2}>\mathrm{H}_{2}>$ He. Blister damage caused by hydrogen was moderate compared to that by nitrogen but more serious than that by helium. With hydrogen and helium, blister size peaked 360 to 900 seconds after decompression, but the blister size continued to increase with nitrogen gas even 3,600 seconds after decompression.

Figure 5 shows the schematic illustration of the effects of gas species on blister size. For blisters forming after decompression, the internal pressure $\Pi$ acts proportionately to the concentration of gas $c$ dissolved in the rubber around the blister. Taking the solubility coefficient to be $S$, the internal pressure $\Pi$, gas concentration $c$ and exposure pressure $p$ can be 
expressed by the following equation.

$$
\Pi=\frac{c}{S} \leq p
$$

This means that the slower the desorption of the dissolved gas molecule, a higher internal pressure $\Pi$ will work on the blister over a longer time after decompression. Unlike $\mathrm{H}_{2}$ and $\mathrm{He}$, with $\mathrm{N}_{2}$, as the gas

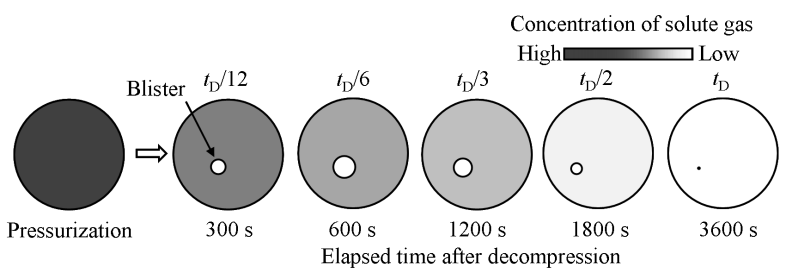

(a) Hydrogen gas $\left(t_{\mathrm{D}}=3600 \mathrm{~s}\right)$

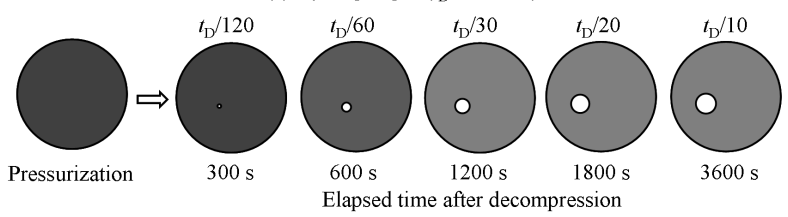

(b) Nitrogen gas $\left(t_{\mathrm{D}}=36000 \mathrm{~s}\right)$

Fig. 5 Schematic illustration of difference in blister initiation behavior of EPDM tested in gas species at $10 \mathrm{MPa}$ under $25^{\circ} \mathrm{C}$ : (a) Hydrogen gas, (b) Nitrogen gas

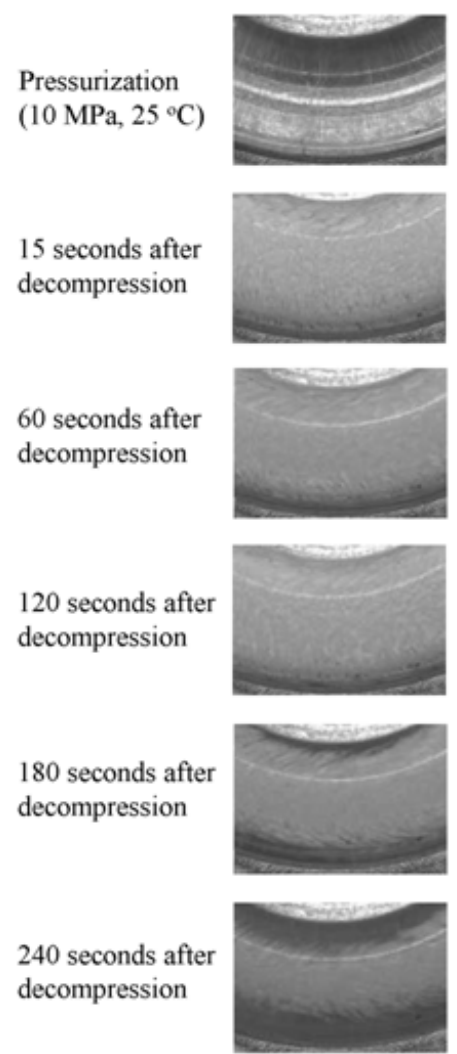

(a) Hydrogen gas diffusion coefficient is small, the dissolved gas molecules more or less do not desorb even 3,600 seconds after decompression. On the other hand, it is known that the rubber shows a time-dependent crack growth under a constant loading, which is generally termed a static crack growth [9]. Thus, the longer the load time, the greater will the crack growth amount be. Due to this reason, the blister initiation behavior depends on the gas diffusion coefficient. Consequently, as shown in Fig. 3, the smaller the diffusion coefficient of the gas is, the more intense the blister initiation behavior of EPDM will be.

\subsubsection{VMQ}

Figure 6 shows the results of observation of the insides of O-rings during pressurization and after decompression of VMQ exposed to hydrogen, helium, and nitrogen at a pressure of $10 \mathrm{MPa}$ under an optical microscope. Regardless of the gas species, the insides of the O-rings after decompression for VMQ differed completely from EPDM. With VMQ, regardless of the gas species, the blisters seen with EPDM did not occur. Only the change in color of the O-rings to white was seen, but they became transparent again with the desorption of the dissolved gas. When the cross-section of the O-rings was observed under an optical microscope before exposure and after the test, no cracks were found, and no marked differences were indicated.
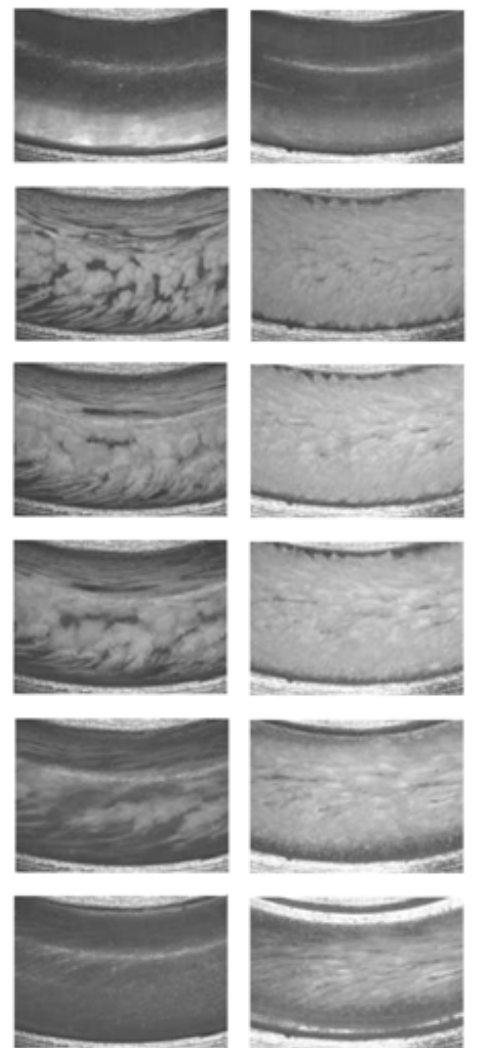

(b) Helium gas

(c) Nitrogen gas

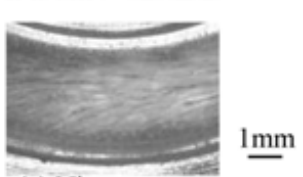

Fig. 6 Blister initiation behavior of VMQ with high-pressure gas decompressions at $10 \mathrm{MPa}$ under $25^{\circ} \mathrm{C}$ : (a) Hydrogen gas, (b) Helium gas, (c) Nitrogen gas 


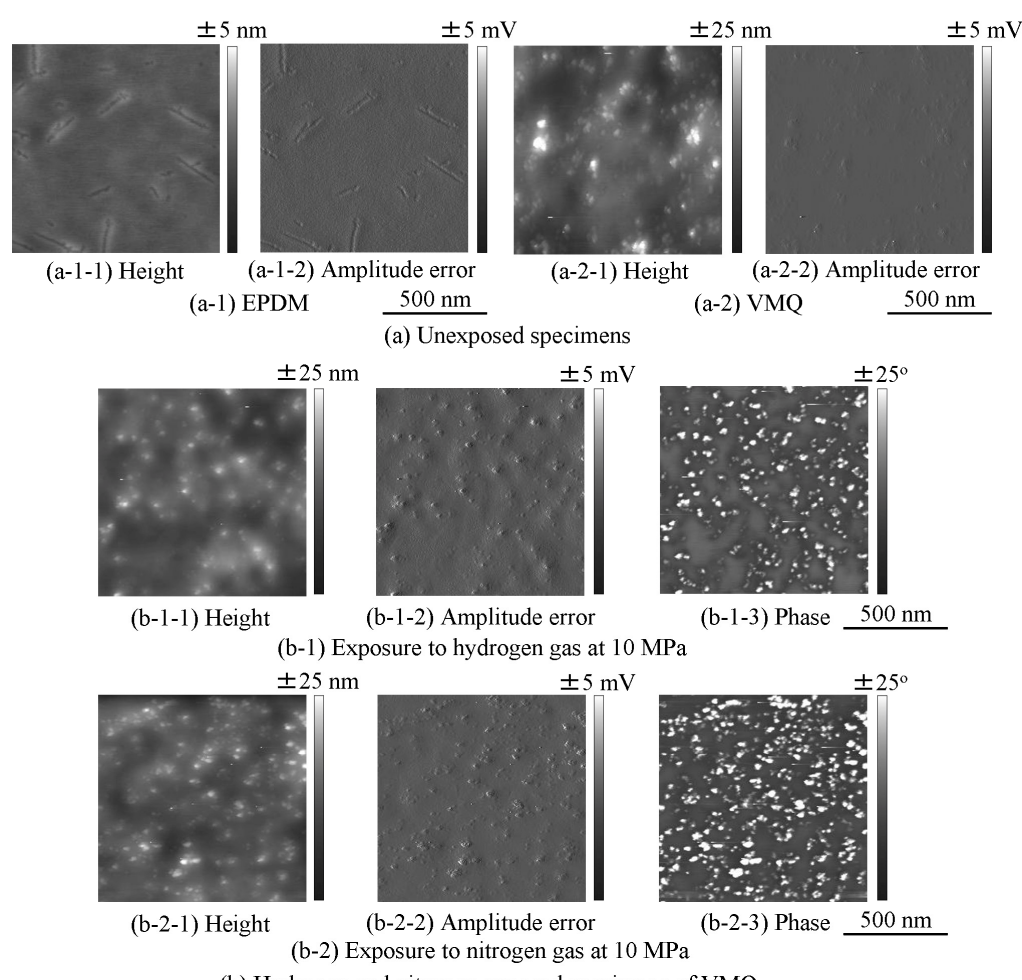

(b) Hydrogen and nitrogen-exposed specimens of VMQ

Fig. 7 AFM images of EPDM and VMQ: (a) Unexposed EPDM and VMQ, (a-1) EPDM, (a-2) VMQ; (b) Hydrogen and nitrogen-exposed specimen of VMQ, (b-1) Exposure to hydrogen gas at $1 \mathrm{MPa},(\mathrm{b}-2)$ Exposure to nitrogen gas at $10 \mathrm{MPa}$

\subsection{Blister initiation process of EPDM and VMQ}

One simple method of estimating the blister initiation limit pressure is the stress instability method developed by Gent et al [10]. With this theory, the constitutive relation of rubber is approximated by the neo-Hookean solid which can be expressed simply by equation (2)

$$
\sigma_{\mathrm{n}}=\frac{E}{3}\left(\lambda-\frac{1}{\lambda^{2}}\right)
$$

where $\sigma_{\mathrm{n}}$ is the nominal stress (first Piola-Kirchhoff stress), $E$ is Young's modulus, and $\lambda$ is the stretch ratio. Considering a spherical cavity with radius $a_{0}$ in an infinite body, and this radius becomes $a$ when the cavity is subject to internal pressure $\Pi$. Based on finite strain theory of elasticity [11], the relationship between the internal pressure $\Pi$ and the tangential stretch at the cavity surface, $a / a_{0}\left(=\lambda_{\mathrm{a}}\right)$, can be expressed for the neo-Hookean solid by equation (3).

$$
\Pi=\frac{E}{6}\left(5-\frac{4}{\lambda_{\mathrm{a}}}-\frac{1}{\lambda_{\mathrm{a}}^{4}}\right)
$$

From the blister initiation limit condition $\left(\lambda_{\mathrm{a}} \rightarrow \infty\right)$, equation (4) can be obtained.

$$
\Pi_{\mathrm{F}} \cong \frac{5 E}{6}
$$

Equation (4) indicates that if spherical gas bubbles existed, which might serve as the starting point of blister initiation in the rubber, the initiation limit pressure $\Pi$ of the blister from this gas bubble should be in the order of the Young's modulus. The Young's modulus of EPDM and VMQ used in this study was the same as $2 \mathrm{MPa}$ or less. According to equation (4), under the conditions of this study (pressure of $10 \mathrm{MPa}$ ), blisters would occur for both EPDM and VMQ. However, no blister occurrence was seen with VMQ. One reason for this may be because VMQ has a greater diffusion coefficient than EPDM (see Fig. 2), which causes the dissolved gas to desorb before blisters occur. However, because the He gas diffusion coefficient of EPDM and the $\mathrm{N}_{2}$ gas diffusion coefficient of VMQ are more or less the same, the differences in the blister initiation state of EPDM and VMQ cannot be explained with equation (4) and Fig. 5.

Yamabe et al. [6] report that there exists a low strength area of sub-micron-size (several $100 \mathrm{~nm}$ ) with EPDM, and micron-size gas bubbles forming and growing from this area after decompression become the starting point of blister initiation, like micron-size defects (see Fig. 8). One reason why blisters do not occur with VMQ was considered to be due to the difference in this formation and growth process of the gas bubbles. We therefore used an atomic force microscope to observe the sub-micron level structures of EPDM and VMQ.

Figure 7(a) shows the AFM images (Height and Amplitude Error images) of EPDM and VMQ which have not been exposed to gas. Like the results of 


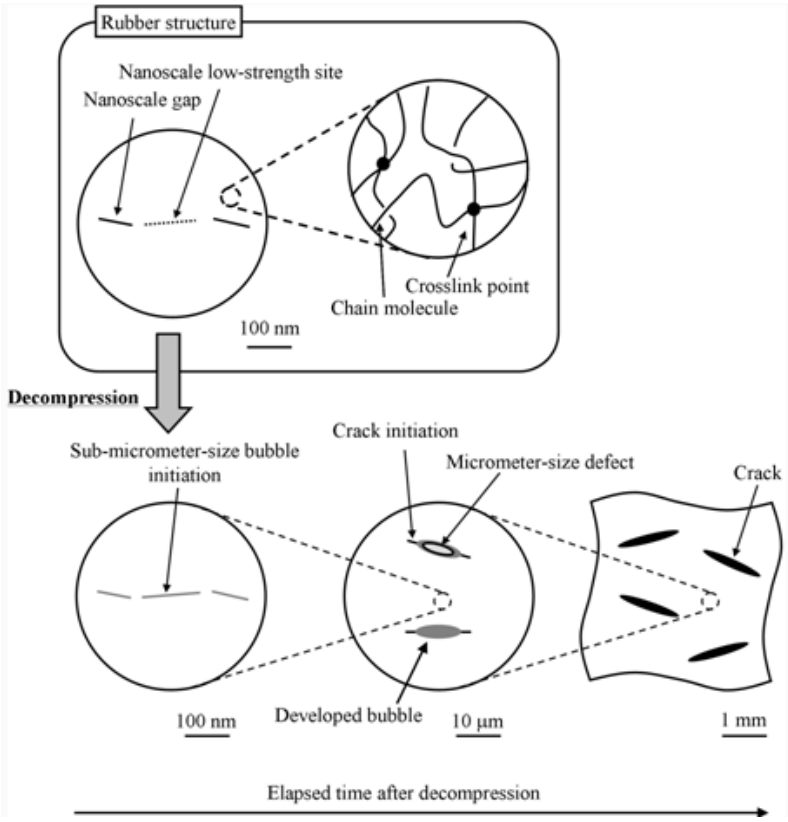

Fig. 8 Schematic illustration of process of blister fracture of EPDM

Yamabe et al. [6], several $100 \mathrm{~nm}$ nanoscale gaps were found in EPDM even though it had not been exposed to gas. On the other hand, no nanoscale gaps were observed for VMQ, only the scattering of several $10 \mathrm{~nm}$ silica in the rubber matrix was seen. According to the fracture process [12] proposed in Fig. 8, gas bubbles which had grown to micron-size from low strength areas of several $100 \mathrm{~nm}$ are thought to become the starting point of blister initiation, like micron-size defects.

Figure 7(b) shows the AFM images (height, amplitude error, phase images) of VMQ exposed to hydrogen and nitrogen. The AFM images of VMQ exposed to hydrogen and nitrogen are similar and show no changes from unexposed material. Although it cannot be asserted that the several $100 \mathrm{~nm}$ nanoscale gaps seen with EPDM in the AFM images of Fig. 7 do not exist in VMQ due to the influence of microscopic roughness of the surface caused by silica, it is at least certain that the rubber matrix of VMQ is more even than EPDM. For this reason, micron-size bubbles are difficult to form and grow with VMQ as compared to EPDM. Only change in color of the rubber to white as seen in Fig. 6 was thought to have occurred, and no blisters were thought to have occurred. Such differences in the microheterogeneous structure may be one of the reasons for the large fracture growth of EPDM in contrast to VMQ.

\section{Conclusion}

In this study, to visualize blisters using an optical microscope (internal blister), transparent EPDM and VMQ O-rings cured with peroxides were exposed to hydrogen, helium, and nitrogen at a pressure of $10 \mathrm{MPa}$, and the effects of the type of polymers and gases on gas permeation characteristics and blister initiation were reviewed. To clarify the effects of rubber structure on blister initiation, submicron-size rubber structures were observed under an atomic force microscope. As a result, it was found that blisters occur regardless of the gas species for EPDM, and the blister size is greater for gases with smaller gas diffusion coefficient. On the other hand, with $\mathrm{VMQ}$, only the change in the color of rubber to white due to the gas species was seen. No blisters were found. AFM observations of EPDM and VMQ revealed low strength areas of several $100 \mathrm{~nm}$ with EPDM due to its microheterogeneous structure, which was not found with VMQ. This microheterogeneous structure is thought to affect the blister initiation of EPDM.

\section{Acknowledgements}

This research is supported by the NEDO project "Fundamental Research Project on Advanced Hydrogen Science (2006-2012).”

\section{References}

[1] Briscoe, B. J., Savvas, T. and Kelly, C. T., "Explosive Decompression Failure of Rubbers - A Review of the Origins of Pneumatic Stress-Induced Rupture in Elastomers," Rubber Chemistry and Technology, 67, 1994, 384-416.

[2] Stevenson, A. and Morgan, G. J., "Fracture of Elastomers by Gas Decompression," Rubber Chemistry and Technology, 68, 1995, 197-211.

[3] Briscoe, B. J. and Zakaria, S., "Sorption and Dilation of Silicone Elastomer Composites at High Gas Pressures: The Role of Interfacial Quality," Journal of Polymer Science Part B: Polymer Physics, 30, 1992, 959-969.

[4] Yamabe, J. and Nishimura, S., "Influence of Fillers on Hydrogen Penetration Properties and Blister Fracture of Rubber Composites for O-Ring Exposed to High-Pressure Hydrogen Gas," International Journal of Hydrogen Energy, 34, 4, 2009, 1977-1989.

[5] Yamabe, J., Koga, A. and Nishimura, S., "A Study on Sealing Behavior of Rubber O-Ring in High Pressure Hydrogen Gas," SAE International Journal of Materials and Manufacturing, 2, 2009, 452-460.

[6] Yamabe, J. and Nishimura, S., "Nanoscale Fracture Analysis by Atomic Force Microscopy of EPDM Rubber due to High-Pressure Hydrogen Decompression," Journal of Materials Science, 46, 2011, 2300-2307.

[7] Van Amerongen, G. J., "Diffusion in Elastomers," Rubber Chemistry and Technology, 37, 5, 1964, 1067-1103.

[8] Yamabe, J., Koga, A. and Nishimura, S., "Fracture Behavior and Hydrogen Permeation Properties of EPDM for Sealing under High Pressure Hydrogen 
Gas,” Nippon Gomu Kyokaishi, 83, 2010, 159-166 (in Japanese).

[9] Lake, G. J. and Lindley, P. B., "Cut Growth and Fatigue of Rubbers. II. Experiments on a Noncrystallizing Rubber," Journal of Applied Polymer Science, 8, 2, 1964, 707-721.

[10] Gent, A. N. and Tompkins, D. A., "Nucleation and Growth of Gas Bubbles in Elastomers," Journal of Applied Physics, 40, 6, 1969, 2520-2525.
[11] Green, A. E. and Zerna, W., "Theoretical Elasticity," Oxford, Clarendon Press, 1954, 104-108.

[12] Yamabe, J. and Nishimura, S., "Investigation of Mechanism of Internal Crack Initiation of Sealing Rubber for High-Pressure Hydrogen Gas," Proceedings of JCCM-2, 2011 (in Japanese). 